\title{
L-CBM signaling in lymphocyte development and function
}

This article was published in the following Dove Press journal:

Journal of Blood Medicine

4 June 2010

Number of times this article has been viewed

\section{Hiromitsu Hara \\ Eiichi Ilzasa \\ Mako Nakaya \\ Hiroki Yoshida}

Department of Biomolecular Sciences, Faculty of Medicine, Saga University, Saga, Japan
Correspondence: Hiromitsu Hara Department of Biomolecular Sciences, Faculty of Medicine, Saga University, 5-I-I Nabeshima, Saga 849-850 I, Japan

Email harah@cc.saga-u.ac.jp
Abstract: The nuclear factor- $\kappa \mathrm{B}(\mathrm{NF}-\kappa \mathrm{B})$ plays a central role in the activation and survival of lymphocytes. NF- $\kappa \mathrm{B}$, therefore, is pivotal for acquired immunity, but the dysregulation of NF- $\mathrm{KB}$ signaling leads to inflammatory diseases and lymphomagenesis. Accumulating evidence has demonstrated that the mucosa-associated lymphoid tissue (MALT) lymphomarelated molecules, B-cell lymphoma 10 (BCL10) and MALT-lymphoma-translocation gene1 (MALT1), are essential signaling components for NF- $\mathrm{\kappa B}$ and mitogen-activated protein kinase (MAPK) activation, mediated by the immunoreceptor tyrosine-based activation motif (ITAM)coupled receptors involved in both innate and adaptive immunity. CARMA1 (also referred to as CARD11 and Bimp3) is a crucial regulator for ITAM-mediated signaling as it forms a complex with BCL10-MALT1 in lymphoid lineage cells such as T, B, natural killer (NK), and natural killer T (NKT) cells, known as the lymphoid CARMA1-BCL10-MALT1 (L-CBM) complex. In this review, recent understanding of the molecular and biological functions and the signal regulation mechanisms of the L-CBM complex are described and its role in disease development and potential as a therapeutic target is further discussed.

Keywords: lymphocytes, inflammatory disease, immunity L-CBM complex

\section{CARMAI-BCLI 0-MALTI (CBM) complexes}

Immunoreceptors such as $\mathrm{T}$ cell receptors (TCRs), B cell receptors (BCRs), Fc receptors (FcRs), activating natural killer cell receptors (NKRs), as well as multiple immunoglobulin (Ig)- or C-type lectin-family receptors expressed on myeloid cells, transduce activation signals by associating with signaling chains (eg, CD3s, $\operatorname{Ig} \alpha, \operatorname{Ig} \beta$, FcR $\gamma$, and DAP12) containing the immunoreceptor tyrosine-based activation motifs (ITAMs), bearing a consensus sequence of YxxL-x6-8-YxxL (where $\mathrm{x}$ denotes any amino acids), in their cytoplasmic domains. ${ }^{1}$ Upon engagement of these ITAM-coupled receptors, an activation signal cascade is initiated with phosphorylation of specific tyrosines in ITAMs by the Src family of kinases. ${ }^{2}$ The phosphorylated ITAMs recruit the tyrosine kinase Syk or zeta-associated protein, $70 \mathrm{kDa}$ (ZAP-70), which bind via the tandem Src homology domain 2 (SH2) and initiate a downstream signaling cascade leading to activation of transcription factors such as nuclear factor- $\kappa \mathrm{B}(\mathrm{NF}-\kappa \mathrm{B})$, nuclear factor activated T-cells (NFAT), and activating protein-1 (AP-1).

The NF- $\kappa \mathrm{B}$ family of transcription factors plays a critical role in immune cell functions such as immune regulation, inflammation, cell survival, and cell cycle progression. ${ }^{3}$ Accumulating evidence has revealed that the mucosa-associated lymphoid tissue (MALT) lymphoma-related molecules, B-cell lymphoma 10 (BCL10) and MALT-lymphoma-translocation gene1 (MALT1), form a complex and play an essential role in NF- $\kappa$ B activation submit your manuscript | www.dovepress.com

Dovepress

9772 
through all of the ITAM-coupled receptors expressed by immune cells, as well as some G-protein-coupled receptors expressed on nonimmune cells. ${ }^{4}$ Recent genetic-based studies have identified the two caspase recruitment domain (CARD)-family adaptor molecules, CARMA1 (also known as CARD11 and Bimp3) and CARD9, regulate the BCL10-MALT1-mediated NF- $\kappa$ B, as well as mitogen-activated protein kinase (MAPK) activation signaling in a cell-type-specific, nonredundant manner. CARMA1 acts in lymphoid cells such as T/B lymphocytes and natural killer (NK) cells whereas CARD9 acts in myeloid cells such as macrophages and dendritic cells (DCs), ${ }^{5-7}$ and thus play a crucial role in both innate and adaptive immune responses. Based on their cell specificity, the CARMA1BCL10-MALT1 and CARD9-BCL10-MALT1 complexes are referred to as the lymphoid CARMA1-BCL10-MALT1 (L-CBM) complex and the myeloid CARD9-BCL10-MALT1 (M-CBM) complex, respectively (Figure 1). ${ }^{8}$ In this article, the role of L-CBM-mediated signaling controlled by CARMA1, with particular emphasis on its regulation for NF- $\kappa B$ activation, in lymphocyte development and activation, as well as in disease, has been reviewed.

\section{L-CBM signaling in T cells}

BCL10 is a member of the CARD family of proteins and associates with Ig-like domains of MALT1 through its serine/threonine
(S/T)-rich domain (Figure 1). When overexpressed, this interaction synergistically induces NF- $\mathrm{KB}$ activation. CARMA1 is a member of CARD-containing membrane-associated guanylate kinase (MAGUK) family and was found to be a binding partner of BCL10 via CARD-CARD interaction (Figure 1), similar to CARD9 and the other members of the CARD-MAGUKs: CARMA2 (CARD14/Bimp2), and CARMA3 (CARD10/ Bimp1). ${ }^{9,10}$ It is thought that the BCL10-MALT1 complex binds to CARMA1 following antigen receptor-triggering to form the L-CBM complex. An association of CARMA1 with MALT1 and BCL10 via a coiled-coil (C-C) domain was also reported, which likely contributes to stabilizing the L-CBM complex (Figure 4). ${ }^{11,12}$ Genetic studies using knockout mice of CARMA1, BCL10, and MALT1, as well as a CARMA1deficient Jurkat cell line, have demonstrated the physiological relevance of the L-CBM complex in TCR and BCR signaling. The T cell phenotype of CARMA1-deficent $\left(C A R D 11^{-/-}\right)$mice closely resembles that of BCL10-deficient $\left(B C L 10^{-/-}\right)$and MALT1-deficient $\left(M A L T 1^{-/-}\right)$mice. ${ }^{13-16}$

Peripheral mature $\mathrm{T}$ cells from these L-CBM-deficient mice show almost complete abrogation of proliferation and cytokine production upon TCR stimulation. As a result, these mice exhibit impaired T cell immunity. ${ }^{13,15}$ Loss of L-CBM molecules abrogates TCR-induced NF- $\mathrm{KB}$ activation owing to defective activation of an inhibitor of $\kappa \mathrm{B}(\mathrm{I} \kappa \mathrm{B})$ kinase (IKK)

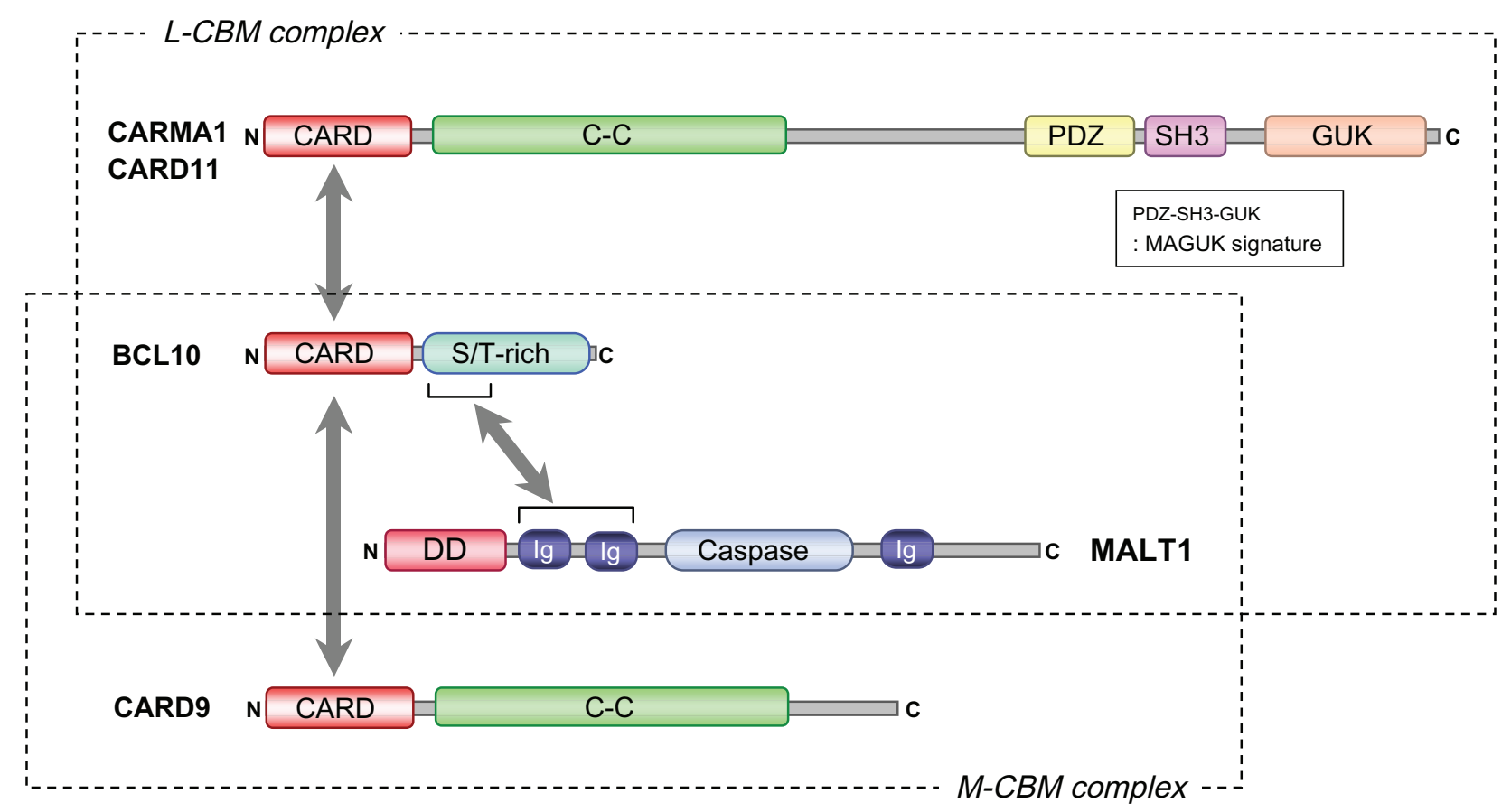

Figure I L-CBM complex and M-CBM complex.

Notes: Structure of CARMAI, CARD9, BCLIO and MALTI are shown.

Abbreviations: L-CBM, lymphoid-CARMAI-BCLI0-MALTI; M-CBM, myeloid-CARD9-BCLI0-MALTI; CARD, caspase recruitment domain; C-C, coiled-coil domain; PRD, protein-kinase-C-regulated domain; PDZ, PSD95, DLGA and ZOI homology domain; SH3, Src-homology 3 domain; GUK, guanylate kinase domain; MAGUK, membraneassociated guanylate kinase; S/T-rich, serine/threonine-rich domain; DD, death domain; Ig, immunoglobulin-like domain. 
(Figure 2), whereas calcium mobilization and proximal tyrosine phosphorylation are unaffected. ${ }^{13,15}$ In addition, CARMA1 and MALT1 deficiency affects the activation of the MAPK c-Jun-Nterminal kinases (JNK), ${ }^{14,15}$ particularly JNK $2,{ }^{17}$ but not the other MAPKs, Erk and p38 (Figure 2). The defective JNK2 activation results in reduced expression of c-Jun. The MAP3K, transforming growth factor $\beta$ kinase 1 (TAK1) and the MAPK kinase, MKK7, are likely involved in this pathway as upstream kinases of JNK2 because these molecules have been shown to be associated with BCL10 upon TCR stimulation. ${ }^{17}$

L-CBM deficiency did not affect overall development of thymocytes, with normal percentages and numbers of

\section{T cells, B cells}

Microbe infection

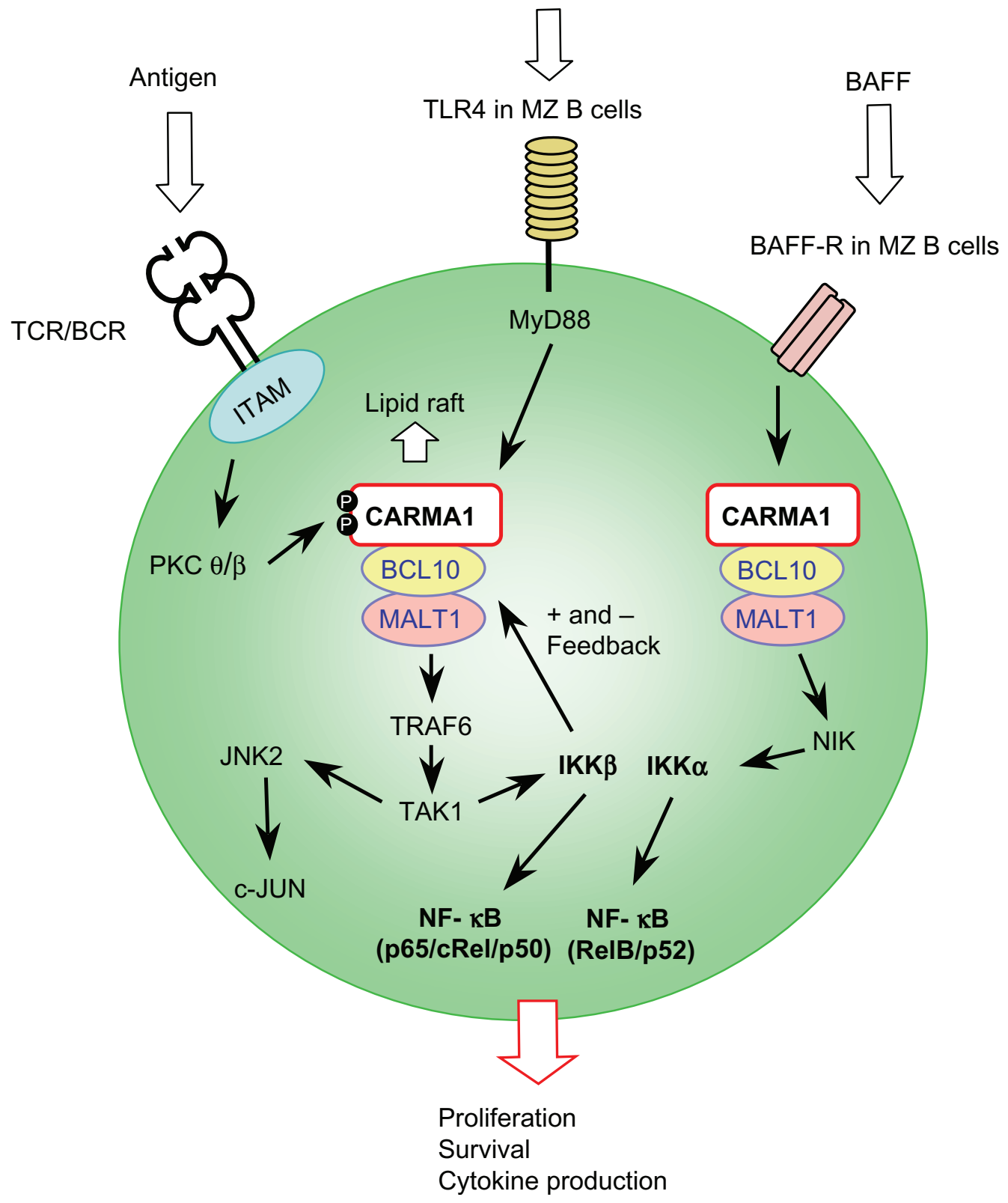

Figure 2 Schematic signaling pathway mediated by the L-CBM complex in T and B lymphocytes.

Notes: The L-CBM complex acts in the canonical NF-KB (p65/p50, cRel/p50) activation pathway mediated by IKK $\beta$ downstream of TCRs and BCRs. L-CBM also controls the JNK, particularly JNK2, activation pathway through antigen receptors, leading to c-JUN activation. Upon antigen receptor stimulation, PKC $\theta$ in T cells and PKC $\beta$ in $B$ cells phosphorylate CARMAI in the PRD region (see Figure 4), enabling the formation and membrane recruitment of the L-CBM complex. IKK $\beta$ positively and negatively regulates L-CBM signaling. These signaling events are regulated by the recruitment of signaling components to specific membrane domains, such as lipid rafts, after receptor triggering. In MZ B cells, L-CBM mediates signals for canonical NF- $\kappa B$ activation through TLR4-Myd88. L-CBM also mediates signals for noncanonical NF- $\kappa B$ (RelB/p52) activation mediated by NIK and IKK $\alpha$ downstream of the BAFF receptor (BAFF-R), which regulates the survival of MZ B cells. 
$\mathrm{CD}^{+} \mathrm{CD}^{+}, \mathrm{CD}^{+} \mathrm{CD} 8^{-}$, and $\mathrm{CD}^{+} \mathrm{CD}^{-}$cells, but increased $\mathrm{CD}^{-}{ }^{-} \mathrm{CD}^{-}{ }^{-}$double negative (DN) cells. ${ }^{13,15}$ This increase may be due to defective thymic egression of a $\mathrm{T}$ cell subpopulation with a DN phenotype rather than impaired early development of conventional T cell precursors, as these DN cells display a mature $\mathrm{T}$ cell phenotype with high levels of CD3 expression. No overt developmental changes in conventional CD4 and CD8 T cells were observed in the peripheral lymphoid organs of mice with L-CBM deficiency; however, the number of naturally occurring regulatory T cells (nTregs) was markedly reduced in the thymus and periphery. ${ }^{18-20}$ This phenotype could likely be attributed to the defective TCR-mediated $\mathrm{NF}-\kappa \mathrm{B}$ signaling, as $\mathrm{T}$ cells deficient for both $\mathrm{PKC} \theta$, the essential upstream kinase of L-CBM, and IKK $\beta$, which controls canonical NF- $\kappa$ B activation pathway through TCRs, also exhibit a similarly significant reduction of nTregs. ${ }^{18} \mathrm{~A}$ study using $C A R D 11^{-/-}$mice revealed that L-CBM controls an early checkpoint in Treg development by enabling the generation of thymic precursors of Tregs. ${ }^{20}$ This deficiency is Tregintrinsic, and overexpression of a constitutive active STAT5, an essential mediator of IL-2/IL-15 receptor signaling, as well as of the antiapoptotic protein, BCL2, failed to rescue this deficiency, indicating that L-CBM neither controls IL-2/IL-15 receptor signaling nor mitochondrion-regulated cell survival during Treg development. Thus, L-CBM-mediated signaling may induce undefined factors essential to commit immature thymocytes to the nTreg lineage, although these factors are dispensable to conventional $\mathrm{T}$ cell development.

\section{L-CBM signaling in B cells}

Reduced follicular (FO) and marginal zone (MZ) B cells in spleen and an almost complete absence of peritoneal B-1 B cells have been consistently observed in $C A R D 11^{-/-}$, $B C L 10^{-/-}$, and $M A L T 1^{-/-}$mice. ${ }^{14,15,21}$ This deficiency of L-CBM molecules results in abrogated BCR-induced NF- $\kappa \mathrm{B}$ activation and thereby defects in $\mathrm{B}$ cell proliferation and survival, although one line of $M A L T 1^{-/-}$mice has exhibited only mild defects in B cell activation through a selective defect in c-Rel activation, which controls B cell survival (Figure 2). ${ }^{14,22}$ CARMA1, and probably BCL10, control JNK activation through BCRs (Figure 2), ${ }^{15}$ whereas MALT1 might be dispensable for it. ${ }^{14,16} \mathrm{CD} 40$-induced activation was also defective in splenic B cells with L-CBM deficiency, ${ }^{13,16,23}$ possibly owing to defective development of MZ B cells, which are the major cells responding to CD40 stimulation. ${ }^{23}$

The involvement of L-CBM in Toll-like receptor (TLR) signaling in B lymphocytes has been suggested, although it remains a controversial issue. $C A R D 11^{-/-}$whole splenic
B cells show impaired proliferation upon TLR4 stimulation, probably due to defective JNK activation. ${ }^{15}$ A similar deficiency was observed in one of the $M A L T 1^{-/}$mouse models. ${ }^{16}$ A study that compared FO and MZ splenic B cells revealed that BCL10 deficiency affected only MZ B cells in response to lipopolysaccharide (LPS) due to impaired NF- $\kappa B$ activation (Figure 2). ${ }^{21}$ The defective LPS response of whole splenic B cells from L-CBM deficient mice might be due to the reduced number and the impaired response of $\mathrm{MZ} B$ cells because MZ B cells have been shown to proliferate much better than FO B cells upon LPS stimulation. ${ }^{24}$

$\mathrm{NF}-\kappa \mathrm{B}$ is activated by either a canonical or a noncanonical pathway. ${ }^{25}$ The canonical pathway, which depends on IKK $\beta$, is activated by engagement of receptors such as TNFR1, IL-1R, TLRs, TCR, and BCR. IKK $\beta$ phosphorylates inhibitors of $\kappa \mathrm{B}$ molecules bound to $\mathrm{NF}-\kappa \mathrm{B}$ subunits such as RelA, allowing NF- $\kappa B$ to translocate to the nucleus. The noncanonical pathway, which depends on IKK $\alpha$, is activated by engagement of receptors for lymphotoxin $\beta$ (LT- $\beta$ ), RANKL, CD40L, and B-cell activation of the tumor necrosis factor (TNF) family (BAFF). IKK $\alpha$ mediates phosphorylation-dependent processing of the NF- $\kappa \mathrm{B} 2$ precursor, p100, resulting in the generation of p52 and its translocation to the nucleus. As discussed so far, L-CBM signaling is crucial for the canonical NF- $\mathrm{KB}$ signaling downstream of TCRs and BCRs. However, recent reports have shown that L-CBM also activates the noncanonical pathway and thereby regulates the survival in $\mathrm{MZ} \mathrm{B}$ cells. ${ }^{26-28}$ E $\mu$-driven human BCL10-transgenic (Tg) mice exhibit constitutive activation of both the canonical and noncanonical NF- $\kappa B$ pathway in splenic B cells. The Tg mice show expanded MZ and B1 B cells and reduced FO and B1a cells, with some older mice developing MZ lymphomas. Lack of MALT1 impairs BAFF-induced phosphorylation and degradation of p100 (Figure 2). The $M A L T 1^{-/-} \mathrm{MZ}$, but not FO B cells, exhibit reduced survival and antiapoptotic gene induction in response to BAFF in vitro, likely owing to the elevated expression and defective BAFF-induced downregulation of TRAF3, a negative modulator of the BAFF-induced survival signal, particularly in MZ B cells. The phenotypes of BAFFTg mice, including increased basal serum Ig, MZ B cells, and B1 B cells, spontaneous germinal center formation, and Ig deposition in the kidney, all disappear in the absence of MALT1 and BCL10. In line with these observations, the B cell phenotype of L-CBM-deficient mice resembles that of the BAFF-R- mutant A/WySnJ mice, ${ }^{29}$ suggesting a role for L-CBM complex in driving the nonclassical NF- $\kappa B$ pathway in BAFF-mediated survival of B cells. 


\section{L-CBM signaling in NK cells}

NK cells play as a sentinel in protecting against tumors and intracellular pathogens. NK cell activation is dependant upon multiple germline-encoded activating and inhibitory receptors that recognize specific ligands on target cells. ${ }^{30}$ These are referred to as activating NK cell receptors (NKRs) and inhibitory NKRs, respectively. When activating NKR signaling dominates inhibitory NKR signaling, NK cells attack targets through two defined effector functions: cytotoxicity; and production of proinflammatory cytokines and chemokines. The activating NKRs are ITAM-coupled receptors and are similar to those in myeloid cells with respect to structure and signaling mechanisms; they belong to either the Ig or C-type lectin family of receptors and use the same ITAM-containing adaptors, DAP12 and FcR $\gamma$. The role of the L- or M-CBM complex in activating NKR signaling has been examined by using $C A R D 9^{-/-}, C A R D 11^{-/}, B C L 10^{-/}$, and $M_{A L T 1^{-/}}$mice. ${ }^{6,731}$ Despite its essential role in myeloid DAP12- and FcR $\gamma$-associated receptors, CARD9 is not required for activation through activating NKRs. ${ }^{6,7}$ Instead, studies have revealed that CARMA1, BCL10, and MALT1 were essential for production of cytokines and chemokines induced by multiple activating NKRs, including Fc $\gamma$ RIII (CD16), NK1.1, Ly49H, Ly49D, and NKG2D, demonstrating that L-CBM, not M-CBM, regulates activation of NKR signaling (Figure 3). However, these deficiencies do not influence either maturation or the repertoire formation of peripheral NK cells. Similar to T and B lymphocytes, the loss of L-CBM impairs NF- $\kappa B$ activation following activation of NKRs (Figure 3). On the other hand, contribution of L-CBM to MAPK activation, particularly JNK and p38, is unclear. In $B C L 10^{-/-}$and $M A L T 1^{-/-} \mathrm{NK}$ cells, JNK and $\mathrm{p} 38$ activation is defective upon stimulation with phorbol myristate acetate plus calcium ionophore $(\mathrm{P} / \mathrm{I})$, which bypasses antigen receptor proximal signaling events by directly activating protein kinase Cs (PKCs); while in CARD $11^{-/} \mathrm{NK}$ cells, all MAPK activation following CD16 crosslinking is normal (Figure 3). Thus, it will be necessary to clarify whether this difference reflects different contributions of CARMA1 and BCL10/ MALT1 in NKR-induced MAPK activation or arises from alternative signaling activation by different stimuli. Interestingly, the cytotoxicity of NK cells induced by activating NKRs is not affected by L-CBM deficiency (Figure 3). This corresponds with normal Vav1 phosphorylation and $\mathrm{Ca}^{2+}$ mobilization, both of which regulate exocytosis of cytotoxic granules, upon activating NKR ligation in $C A R D 11^{-/-} \mathrm{NK}$ cells. ${ }^{6}$ Together, L-CBM controls a distinct function of NK cells induced by NKR triggering (ie, cytokine and chemokine production, but not cytotoxicity) by selectively regulating the NF- $\mathrm{KB}$ activation signal.

\section{Signaling regulation of L-CBM complex}

In this section, we discuss recent advances in the understanding of the molecular mechanisms that regulate L-CBM signaling. Multiple regulation mechanisms; involving phosphorylation, ubiquitylation, oligomerization, caspase activation, and recruitment to plasma membrane have been proposed to control L-CBM function (Figure 4). Upon activation of antigen receptors, CARMA1 and BCL10 are phosphorylated by several kinases. Phosphorylation by some kinases represented by PKCs, positively regulates L-CBM signaling by modifying CARMA1 conformation, enabling it to form a signalosome with BCL10/MALT1 and downstream components for the NF- $\kappa B$ activation pathway. ${ }^{32}$ Phosphorylation by other kinases has been shown to downmodulate L-CBM signaling by interfering with signalosome formation or targeting CARMA1 and BCL10 for degradation. Cascading oligomerization of L-CBM components and downstream molecules is a crucial event for the activation of this pathway. The lysine (K) 63-linked ubiquitination of BCL10 and MALT1 following TCR activation provides docking sites for downstream signaling molecules such as TRAF6 and NEMO, a necessity for the oligomerization cascade leading to IKK activation. By contrast, it has been reported that BCL10 and CARMA1 undergo degradation followed by K48-linked ubiquitination after antigen receptor-induced activation that may work to limit the amplitude of L-CBM signaling. L-CBM complex was shown to interact with caspase- 8 and thereby regulate the caspase-8-c-FLIP ${ }_{\mathrm{L}}$-mediated NF- $\kappa B$ activation pathway. Paracaspase activity of MALT1 was recently reported and the proteplytic activity shown to fine tune L-CBM-mediated NF- $\mathrm{\kappa B}$ signaling. Various lines of evidence suggest that plasma membrane recruitment of $\mathrm{L}-\mathrm{CBM}$ molecules is a crucial event for its function in NF- $\kappa \mathrm{B}$ activation signaling.

\section{Regulation by PKCs}

L-CBM deficiency abrogates NF- $\mathrm{KB}$ activation in T, B and NK cells upon stimulation with $\mathrm{P} / \mathrm{I},{ }^{6,7,13-15}$ indicating that L-CBM acts downstream of PKCs. PKC-deficient mice have confirmed this notion; the deficiency of PKC $\theta$ in $\mathrm{T}$ and $\mathrm{NK}$ cells, and of $\mathrm{PKC} \beta$ in $\mathrm{B}$ cells, resulted in profound defects in TCR-, activating NKR- and BCR-induced NF- $\mathrm{KB}$ activation, respectively. ${ }^{33,34}$ This regulation could be explained by findings that CARMA1 is a direct target of these PKCs. 


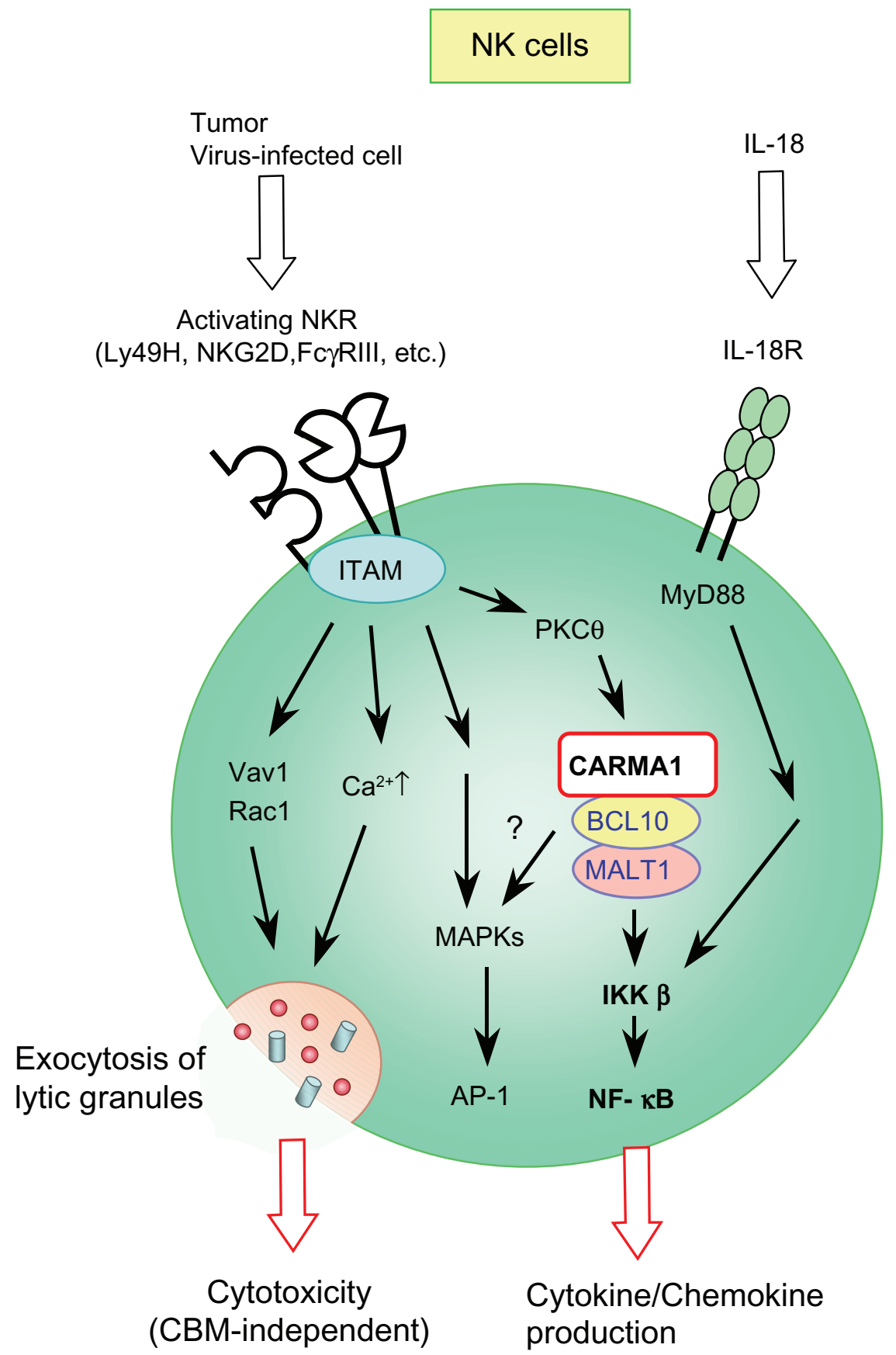

Figure 3 Schematic signaling pathway mediated by the L-CBM complex in NK cells.

Notes: The L-CBM complex acts in the canonical NF-KB activation pathway through activating NK cell receptors (NKR) such as Ly49H, Ly49D, NKI.I, NKG2D, Fc $7 R$ III, etc. L-CBM might regulate MAPK activation through activating NKR, although this notion remains controversial. Similar to T cells, PKC $\theta$ activity is required for NF- $K B$ activation through activating NKR. IL-I8R-MyD88-mediated NF-KB activation does not require L-CBM in NK cells. While the PKC $\theta-L-C B M$ signaling is essential to cytokine/chemokine production, it is not necessary for cytotoxicity.

Upon antigen receptor stimulation, CARMA1 is phosphorylated by $\mathrm{PKC} \theta$ and $\mathrm{PKC} \beta$ in the so called $\mathrm{PKC}$-regulated domain (PRD), ${ }^{32}$ which is the linker sequence between the $\mathrm{C}-\mathrm{C}$ and the PDZ domain (Figure 4). At least three serine residues (S552, S637, and S645 in human CARMA1) have been identified as the target sites. ${ }^{35,36} \mathrm{~A}$ model has been proposed where PRD phosphorylation destabilizes and induces conformational changes of CARMA1 from an inhibitory form to an active one that is accessible to BCL10 and other downstream molecules. ${ }^{11,12,32,37}$ In contrast to this essential PKC-dependent regulation in L-CBM, the fact that CARD9 lacks a PRD suggests that M-CBM-mediated signaling cannot be regulated by PKCs in a similar way to CARMA1. Indeed, lack of CARD9 or BCL10 in bone 


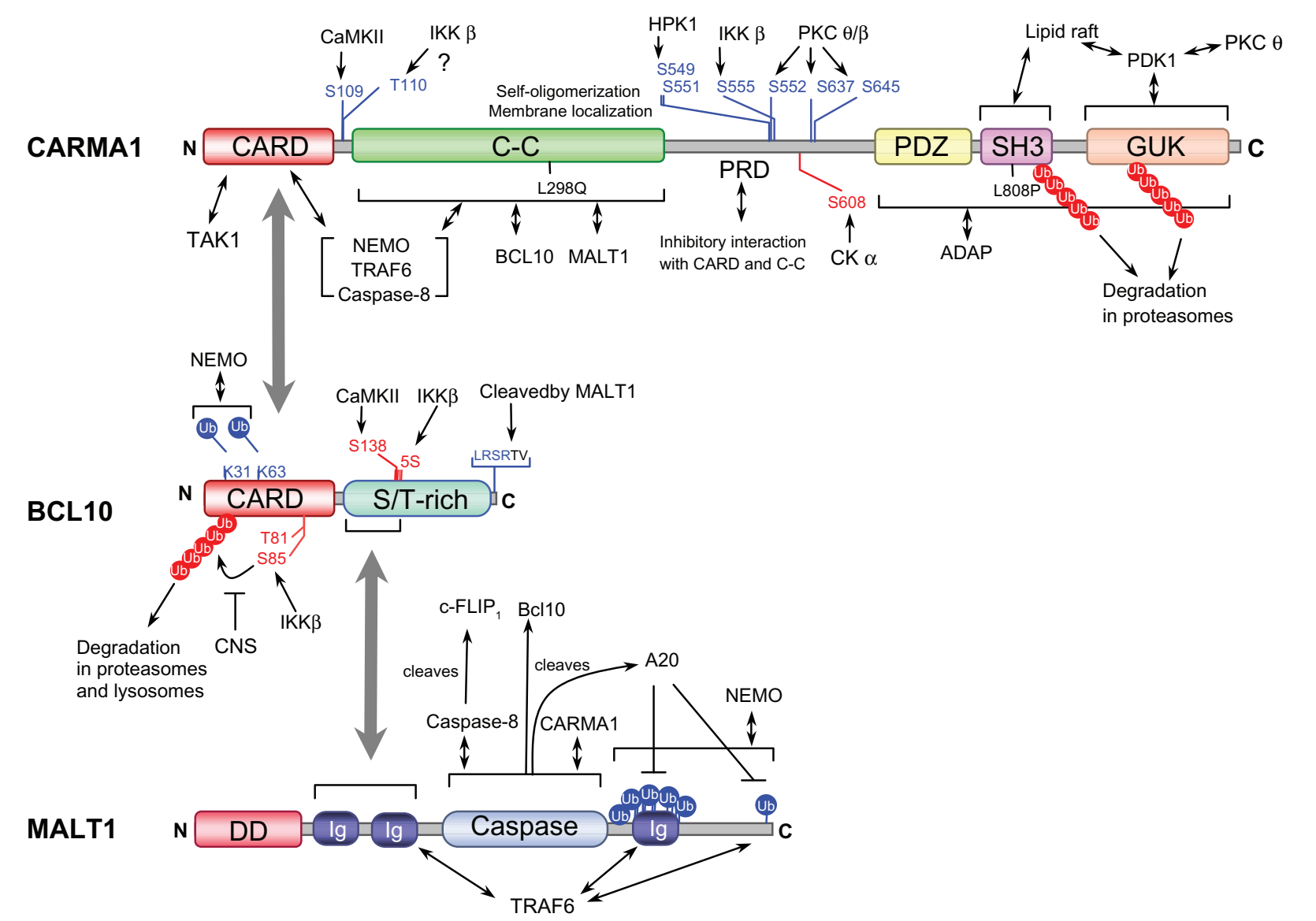

Figure 4 Regulatory phosphorylation sites, polyubiquitylation sites (Ub), proteolytic cleavage sites, and functional domains in L-CBM molecules.

Notes: The figure refers to the human CARMAI, BCLI0, and MALTI protein unless otherwise indicated. Blue text denotes positive regulatory sites and red text denotes negative regulation sites. L298Q (in mouse) and L808P indicate loss-of-function point mutations found in CARMAI.

Abbreviations: PRD, PKC-regulated domain; 5S, five serines; CNS, COP9 signalosome.

marrow-derived dendritic cells (BMDCs) did not affect IKK activation and cytokine production in response to $\mathrm{P} / \mathrm{I} .{ }^{6} \mathrm{In}$ addition, treatment with a PKC inhibitor effectively blocked cytokine production through ITAM-coupled receptors in NK cells, but not in BMDCs. It was so, even when NK cells and BMDCs were activated through the same receptor, CD16. This would indicate that $\mathrm{PKC}$ activation is required for L-CBM-, but not M-CBM-mediated signaling. However, loss of CARMA1 abrogates P/I-induced NF- $\mathrm{\kappa B}$ activation in BMDCs, suggesting that CARMA1 is essential for PKCmediated NF- $\mathrm{\kappa B}$ activation in both lymphoid and myeloid cells, although the PKC-CARMA1 axis in myeloid cells is not utilized downstream of myeloid ITAM receptors, but might be used in signaling through other receptors. In line with this, the overexpression of CARD9 in CARMA1-deficient cells failed to restore $\mathrm{PKC}$-mediated NF- $\mathrm{\kappa B}$ activation. Therefore, these results indicate that L-CBM and M-CBM signaling are regulated by different molecular mechanisms and thus they are functionally not interchangeable.

\section{Regulation by other kinases}

It has been suggested that L-CBM signaling is regulated through phosphorylation by kinases other than PKCs. Calmodulin-dependent protein kinase (CaMKII) phosphorylates CARMA1 on Ser109 after TCR engagement and facilitates the interaction between CARMA1 and BCL10 (Figure 4). ${ }^{38}$ CaMKII also phosphorylates BCL10 on Ser138 but this phosphorylation is involved in the attenuation of NF- $\kappa B$ activation (Figure 4) ${ }^{39}$ Hematopoietic progenitor kinase 1 (HPK1) has been suggested to be involved in the JNK and NF- $\mathrm{KB}$ pathways in TCR signaling and regulates activation and survival of $\mathrm{T}$ cells. ${ }^{40} \mathrm{~A}$ recent report has shown that HPK1 interacts with CARMA1 after TCR stimulation and phosphorylates CARMA1 on Ser549 and Ser551 within the PRD (Figure 4). Although the precise mechanism is unclear, this phosphorylation is critical for TCR-mediated NF- $\mathrm{KB}$ activation and IL-2 production in Jurkat cells. ${ }^{40}$ IKK $\beta$ phosphorylates CARMA1 on Ser578 and probably on Thr119 in chickens (corresponding to 
Ser555 and Thr110 in humans, respectively) upon BCR stimulation (Figure 4). ${ }^{41}$ This phosphorylation is postulated to enhance the assembly of the L-CBM complex, resulting in an increased ability of CARMA1 to activate IKK. Other studies have suggested that IKK $\beta$ phosphorylates BCL10 on five serines within the MALT1-interacting, S/T-rich domain and on Thr81 and Ser55 within CARD, upon TCR stimulation (Figure 4). ${ }^{42,43}$ The former causes disengagement of BCL10 from MALT1 and interferes with IKK ubiquitination and the latter induces BCL10 degradation via the $\beta$-TrCP ubiquitin ligase/proteasome pathway, resulting in negative regulation and attenuation of L-CBM-mediated NF- $\kappa \mathrm{B}$ signaling. Casein kinase (CK) $1 \alpha$ has a contrasting role in L-CBM signaling. $\mathrm{CK} 1 \alpha$ associates with the L-CBM complex through the PRD of CARMA1 after TCR stimulation and participates in NF- $\kappa \mathrm{B}$ activation, proliferation, and cytokine production. However, $\mathrm{CK} 1 \alpha$ kinase activity subsequently contributes to the negative feedback of NF- $\kappa \mathrm{B}$ activation by phosphorylating CARMA1 on Ser608 (Figure 4). ${ }^{44}$

\section{Regulation by oligomerization and ubiquitination}

Oligomerization and its associated ubiquitination events play an important role in L-CBM signaling. A recent study indicated that CARMA1 constitutively oligomerizes via its C-C domain and this self-oligomerization is required for TCR-induced NF- $\kappa \mathrm{B}$ activation (Figure 4$).{ }^{45}$ Similarly, a previous study using mice bearing a point mutation (Leu298Gln) in the C-C domain of CARMA1, which is likely to disrupt C-C domain structure, demonstrated that integrity of the $\mathrm{C}-\mathrm{C}$ domain is crucial for antigen receptor-induced IKK and JNK activation (Figure 4). ${ }^{46}$ CARMA1 forms an active conformation after receptor stimulation, which is followed by the recruitment of downstream molecules, triggering a downstream oligomerization and ubiquitination cascade. ${ }^{32,47}$ BCL10-dependent MALT1 oligomerization induces activation of TNF receptor-associated factor (TRAF) 6, a ubiquitin ligase, which in turn activates the IKK complex through lysine (K) 63-linked ubiquitylation of the regulatory subunit of IKK NEMO (an essential NF- $\kappa B$ modulator). During this process, TRAF6 itself is self-ubiquitinated, enabling recruitment of TAK1 and TAK1-binding protein 2 (TAB2), which then activates IKK $\alpha$ and IKK $\beta$ by phosphorylation. ${ }^{48}$ BCL10 and MALT1 also undergo K63linked ubiquitination in the CARD domain and the C-terminal region, respectively, upon $\mathrm{T}$ cell activation, which is likely to provide a docking surface for the recruitment of NEMO (Figure 4). ${ }^{49,50}$ Interestingly, Zhou et al reported that MALT1 itself has an E3-ligase activity at C-terminus that targets both MALT and NEMO for ubiquitination. ${ }^{51}$

In contrast, signaling inhibition by ubiquitination of L-CBM components has also been reported. CARMA1 is K48-linked-polyubiqutinated in the MAGUK region and degraded by the proteasome after antigen receptor-induced activation in $\mathrm{T}$ and $\mathrm{B}$ cells (Figure 4 ). ${ }^{52}$ The ubiquitinationdependent degradation of CARMA1 requires the phosphorylation of the PRD by PKC. A region between $\mathrm{SH} 3$ and the guanylate kinase domain (GUK), termed the Hook domain, plays a regulatory role in this process. Elimination of the ubiquitination sites from the MAGUK region results in elevated basal and inducible NF- $\mathrm{KB}$ and JNK activation, thus this activation-dependent mechanism likely provides an intrinsic feedback control of L-CBM signaling. In vitro experiments have suggested that the cellular inhibitor of apoptosis protein (cIAP) might target this MAGUK ubiquitination, although a cIAP antagonist that depletes the endogenous pool of cIAP did not affect P/I-induced CARMA1 degradation. Another ubiquitination-mediated negative regulation of CARMA1 has been suggested where Cbl-b-promoted monoubiquitination of CARMA1 is involved in the anergy induction in NKT cells. ${ }^{53}$ It has been shown that BCL10 undergoes degradation following ubiquitination after activation of lymphocytes through antigen receptors, ${ }^{43,54,55}$ resulting in termination of L-CBM signaling (Figure 4). NEDD, cIAP, $\beta$-TrCP, and Itch have been suggested as ubiquitin ligases (E3) of BCL10. Both the proteasomal pathway via $\beta$-TrCP and the lysosomal pathway via NEDD and Itch have been proposed for BCL10 degradation. ${ }^{43,54,55}$ The proteasomal pathway requires the phosphorylation of CARD by IKK $\beta,{ }^{43,54,55}$ while the lysosomal pathway requires intact CARD and the novel PKC activity. ${ }^{43,54,55}$ The COP9 signalosome (CNS), a pleiotropic regulator of the ubiquitin/26S proteasome system, controls antigen responses in T cells. The CNS subunit 5 (CNS5) has been shown to interact with L-CBM in activated T cells. The CNS5, as well as CNS2, fine tunes IKK activation by maintaining BCL10 stability, most likely by interfering with the polyubiquitination and degradation of BCL10 (Figure 4). ${ }^{56}$ The deubiquitinating enzyme, A20, has been shown to function as a negative regulator for $\mathrm{T}$ cell activation. ${ }^{57}$ Duwel et al have recently reported that A20 catalyzes the removal of the K63-linked ubiquitin chains attached to MALT1 and therefore regulates duration and strength of IKK activity (Figure 4). ${ }^{58}$

\section{Regulation by caspases}

MALT1 was originally identified as a caspase homolog named paracaspase. ${ }^{59}$ Recent studies have revealed that the paracaspase domain of MALT1 possesses protease 
activity, cleaving BCL10, ${ }^{60}$ and the NF- $\mathrm{\kappa B}$ inhibitor, A20 ${ }^{61}$ (Figure 4). Although the protease activity is required for optimal activation of NF- $\mathrm{KB}$ following TCR ligation, the MALT1-dependent BCL10 cleavage is not required for activation of NF- $\mathrm{KB}$ and $\mathrm{JNK}$. Instead, it is necessary for TCR-induced cell adhesion to the extracellular matrix protein fibronectin. In contrast to other cells, lymphoid cells, particularly T cells, constitutively express A20. The cleavage of A20 by MALT1 disrupts its inhibitory effect on TCR-induced NF- $\mathrm{KB}$ activation and IL-2 production. The proteolytic activity of MALT1, therefore, is likely to act as a fine tuner in L-CBM signaling. This activity of MALT1 might not to be involved in M-CBM signaling because BCL10 cleavage was not detected when THP-1 monocytic cells were stimulated through ITAM-coupled receptors. ${ }^{60}$

Besides its established role in lymphocyte apoptosis, caspase- 8 and its proteolytically inactive homolog, c-FLIP ${ }_{L}$, are known to be essential for lymphocyte activation in both mice and humans, ${ }^{62-64}$ probably by interacting with the L-CBM complex and regulating NF- $\kappa B$ activation. A study has shown that MALT1 directly associates with procaspase- 8 and induces limited autoprocessing of procaspase- 8 (Figure 4), ${ }^{65}$ which generates an active form of caspase- 8 that lacks the capacity to activate apoptotic substrates including caspase-3, but retains activity toward c-FLIP ${ }_{L}$ to generate p43-FLIP. The cleaved form of c-FLIP ${ }_{L}$ can strongly induce activation of NF- $\kappa \mathrm{B}$ signaling pathway through interaction with TRAF $2{ }^{66}$ The association of caspase- 8 with CARD and the C-C domain of the 'active' form of CARMA1 has also been reported (Figure 4$){ }^{12}$

\section{Regulation by membrane recruitment}

Membrane recruitment is a crucial event in L-CBM-mediated $\mathrm{NF}-\mathrm{KB}$ activation. It has been reported that BCL10, PKC $\theta$, PKC $\beta$, MALT1, procaspase-8, c-FLIP ${ }_{L}$, and the IKK complex are recruited into lipid rafts after antigen receptor stimulation and that this recruitment is crucial for NF- $\mathrm{KB}$ activation. ${ }^{33,67,68}$ CARMA1 resides in both the cytoplasm and lipid rafts of resting cells, but the amount of CARMA1 in lipid rafts increases after activation. This localization of CARMA1 is of particular importance for L-CBM signaling because mutations such as Leu808Pro mutation (L808P in Figure 4) that disrupt membrane localization of CARMA1 impair TCR-induced NF- $\kappa B$ activation. ${ }^{45,67}$ Genetic studies have demonstrated that CARMA1 is essential for the recruitment of BCL10-MALT1 and IKK complexes to lipid rafts. ${ }^{67,69}$

CARMA1 was also shown to control $\mathrm{PKC} \theta$ recruitment in Jurkat cells. ${ }^{67}$ An SLP-76-binding molecule, adhesion and degranulation-promoting adapter protein (ADAP), which functions in the inside-out signaling for integrin activation, has been shown to be critical for TCR-mediated NF- $\mathrm{KB}$ activation by acting as a link between the TCR-ZAP70-SLP76 signaling complex and L-CBM by binding to CARMA1 (Figure 4). ADAP-deficient T cells revealed impaired L-CBM complex formation in the membrane and defective NF- $\mathrm{KB}$ activation. ${ }^{70}$ It has been shown that 3-phosphoinositidedependent kinase 1 (PDK1) recruits PKC $\theta$ and CARMA1 to lipid rafts upon TCR stimulation (Figure 4) ${ }^{71}$ A recent study using mice with a $\mathrm{T}$ cell-specific deletion of PKD1 has revealed that $\mathrm{CD} 28$ ligation facilitates NF- $\mathrm{\kappa B}$ activation by regulating immune synapse localization and phosphorylation of PDK1, which enables the binding of PKC $\theta$ and CARMA $1 .{ }^{72}$ In contrast to the essential role of PDK1 in TCR-mediated NF- $\kappa B$ activation, the deletion of PDK1 in a chicken B cell line does not alter BCR-mediated NF- $\kappa B$ activation, ${ }^{41}$ indicating a possible difference in the regulation mechanism of L-CBM recruitment to the membrane in TCR- versus BCR-mediated signaling pathways for NF- $\kappa B$ activation.

\section{L-CBM signaling in disease}

Three chromosomal translocations of L-CBM genes, $\mathrm{t}(11 ; 18)$ (q21; 21$), \mathrm{t}(1 ; 14)(\mathrm{p} 22 ; \mathrm{q} 32)$, and $\mathrm{t}(14 ; 18)(\mathrm{q} 32 ; \mathrm{q} 21)$, have been reported and well characterized in MALT lymphoma (MALTL). ${ }^{73}$ The MALT1 gene was originally identified in a break point of $\mathrm{t}(11 ; 18)(\mathrm{q} 21 ; \mathrm{q} 21)$. This translocation generates API2-MALT1 fusion products that comprise the $\mathrm{N}$-terminus of API2 with three intact baculovirus IAP repeat (BIR) domains and the C-terminus of the MALT1 with intact caspase-like domains. The fusion product, but neither API2 nor MALT1 alone, is capable of activating NF- $\mathrm{KB}$. The translocations, $\mathrm{t}(1 ; 14)(\mathrm{p} 22 ; \mathrm{q} 32)$ and $\mathrm{t}(14 ; 18)(\mathrm{q} 32 ; \mathrm{q} 21)$, bring the BCL10 and MALT1 genes under the regulatory control of the Ig heavy chain ( $\mathrm{IgH})$ enhancer, respectively, thereby deregulating its expression and culminating in aberrant NF- $\mathrm{KB}$ activation. In addition to chromosomal translocations, BCL10 gene amplification has been reported in pancreatic cancer and nodal diffuse large B cell lymphoma (DLBCL). ${ }^{74}$ Similarly, MALT1 gene amplification was found in cell lines of marginal zone B cell lymphoma and DLBCL. ${ }^{75,76}$

In contrast to normal B cells that express BCL10 in the cytoplasm, MALT lymphoma cells bearing $\mathrm{t}(11 ; 18)$ $(\mathrm{q} 21 ; \mathrm{q} 21)$ and $\mathrm{t}(1 ; 14)(\mathrm{p} 22 ; \mathrm{q} 32)$ express the protein predominantly in the nucleus. Shen et al reported that $70 \%(28 / 40)$ of nasal NK/T cell lymphoma (NL) cases demonstrated aberrant nuclear staining of BCL10 and concomitant nuclear 
localization of NF- $\mathrm{kB}$, although $\mathrm{t}(11 ; 18)(\mathrm{q} 21 ; \mathrm{q} 21)$ and $\mathrm{t}(1 ; 14)(\mathrm{p} 22 ; \mathrm{q} 32)$ are not present in NLs, indicating a possible relationship between aberrant BCL10 nuclear localization and tumorigenesis. ${ }^{77}$

Among the subtypes of DLBCL, the least curable activated-B-cell-like (ABC) subtype DLBCLs, but not the germinal center B cell-like (GCB) subtype, relies on constitutive NF- $\kappa \mathrm{B}$ signaling for survival. A loss-of-function RNA interference screen for genes required for survival of ABC DLBCL revealed that CARMA1 is a key upstream signaling component responsible for the constitutive IKK activity in ABC DLBCL. ${ }^{78}$ In line with this, oncogenic missense mutations of the CARMA1 gene, all within exons encoding the $\mathrm{C}-\mathrm{C}$, have been found in ABC DLBCL. ${ }^{79}$ These mutations constitutively activate the NF- $\mathrm{KB}$ pathway and enhance antigen receptor signaling to NF- $\mathrm{\kappa B}$, possibly owing to aggregate formation of the mutant proteins. MALT1 is constitutively active in ABC DLBCL, but not GCB DLBCL lines. ${ }^{80}$ The oncogenic forms of CARMA1 are more potent than wild type CARMA1 in inducing proteolytic activity of MALT1. Inhibition of MALT1 activity, with the inhibitor z-VRPR-fmk, specifically affected the growth and survival of ABC DLBCLs. Thus, the MALT1 proteolytic activity might be a promising target for DLBCL therapy.

BCL10-transgenic mice elevate BAFF expression and specifically promote survival of MZ B cells and some mice develop splenic MZ lymphomas (MZL). ${ }^{26}$ It has been reported that BAFF overexpression, with concomitant nuclear expression of BCL10 and NF-KB activation, is associated with Helicobacter pylori-independent growth of gastric DLBCL with histologic evidence of MALT lymphoma. ${ }^{81}$ Thus, L-CBM-regulated BAFF signaling and vice versa might contribute to the development of MALTL, MZL, and DLBCL.

L-CBM, therefore, is an attractive therapeutic target for B-cell lymphomas and diseases associated with aberrant lymphocyte activation. However, further elucidation of L-CBM signaling is required for such applications, especially in approaches utilizing suppression of L-CBM activity. Mice with the Leu298Gln (L298Q in Figure 4) point mutation in CARMA1 exhibit profound defects in humoral immune response, whereas, paradoxically, the mice develop a hyperIgE syndrome with atopic manifestations as they age. ${ }^{46}$ This indicates that the amplitude of L-CBM signaling balances immunity versus tolerance and is thereby involved in the maintenance of immune homeostasis.

\section{Disclosures}

The authors report no conflicts of interest in this work.

\section{References}

1. Isakov N. Role of immunoreceptor tyrosine-based activation motif in signal transduction from antigen and Fc receptors. Adv Immunol. 1998;69:183-247.

2. Samelson LE. Signal transduction mediated by the T cell antigen receptor: the role of adapter proteins. Annu Rev Immunol. 2002;20:371-394

3. Li Q, Verma IM. NF-אB regulation in the immune system. Nat Rev Immunol. 2002;2:725-734.

4. Wegener E, Krappmann D. CARD-Bcl10-Malt1 signalosomes: missing link to NF-kB. Sci STKE. 2007;2007:pe21.

5. Hara $\mathrm{H}$, Ishihara $\mathrm{C}$, Takeuchi $\mathrm{A}$, et al. The adaptor protein CARD9 is essential for the activation of myeloid cells through ITAM-associated and Toll-like receptors. Nat Immunol. 2007;8:619-629.

6. Hara H, Ishihara $\mathrm{C}$, Takeuchi A, et al. Cell type-specific regulation of ITAM-mediated NF- $\mathrm{KB}$ activation by the adaptors, CARMA1 and CARD9. J Immunol. 2008;181:918-930.

7. Gross O, Grupp C, Steinberg C, et al. Multiple ITAM-coupled NK-cell receptors engage the Bcl10/Malt1 complex via Carmal for NF- $\mathrm{\kappa B}$ and MAPK activation to selectively control cytokine production. Blood. 2008; 112:2421-2428.

8. Hara H, Saito T. CARD9 versus CARMA1 in innate and adaptive immunity. Trends Immunol. 2009;30:234-242.

9. Thome M. CARMA1, BCL-10 and MALT1 in lymphocyte development and activation. Nat Rev Immunol. 2004;4:348-359.

10. Bertin J, Guo Y, Wang L, et al. CARD9 is a novel caspase recruitment domain-containing protein that interacts with BCL10/CLAP and activates NF-кB. J Biol Chem. 2000;275:41082-41086.

11. Che T, You Y, Wang D, Tanner MJ, Dixit VM, Lin X. MALT1/paracaspase is a signaling component downstream of CARMA1 and mediates $\mathrm{T}$ cell receptor-induced NF- $\mathrm{\kappa B}$ activation. $J$ Biol Chem. 2004;279:15870-15876.

12. McCully RR, Pomerantz JL. The protein kinase C-responsive inhibitory domain of CARD11 functions in NF- $\mathrm{KB}$ activation to regulate the association of multiple signaling cofactors that differentially depend on Bcl10 and MALT1 for association. Mol Cell Biol. 2008;28:5668-5686.

13. Ruland J, Duncan GS, Elia A, et al. Bcl10 is a positive regulator of antigen receptor-induced activation of $\mathrm{NF}-\mathrm{KB}$ and neural tube closure. Cell. 2001;104:33-42.

14. Ruland J, Duncan GS, Wakeham A, Mak TW. Differential requirement for Malt1 in $\mathrm{T}$ and $\mathrm{B}$ cell antigen receptor signaling. Immunity. 2003;19:749-758.

15. Hara $\mathrm{H}$, Wada $\mathrm{T}$, Bakal $\mathrm{C}$, et al. The MAGUK family protein CARD11 is essential for lymphocyte activation. Immunity. 2003;18:763-775.

16. Ruefli-Brasse AA, French DM, Dixit VM. Regulation of NF-KBdependent lymphocyte activation and development by paracaspase. Science. 2003;302:1581-1584.

17. Blonska M, Pappu BP, Matsumoto R, et al. The CARMA1-Bcl10 signaling complex selectively regulates JNK2 kinase in the $\mathrm{T}$ cell receptor-signaling pathway. Immunity. 2007;26:55-66.

18. Schmidt-Supprian M, Tian J, Grant EP, et al. Differential dependence of CD4+CD25+ regulatory and natural killer-like T cells on signals leading to NF-KB activation. Proc Natl Acad Sci U S A. 2004;101:4566-4571.

19. Medoff BD, Sandall BP, Landry A, et al. Differential requirement for CARMA1 in agonist-selected T-cell development. Eur J Immunol. 2009;39:78-84.

20. Molinero LL, Yang J, Gajewski T, Abraham C, Farrar MA, Alegre ML. CARMA1 controls an early checkpoint in the thymic development of FoxP3+ regulatory T cells. J Immunol. 2009;182:6736-6743.

21. Xue L, Morris SW, Orihuela C, et al. Defective development and function of Bcl10-deficient follicular, marginal zone and B1 B cells. Nat Immunol. 2003;4:857-865.

22. Ferch U, zum Buschenfelde CM, Gewies A, et al. MALT1 directs B cell receptor-induced canonical nuclear factor- $\mathrm{\kappa B}$ signaling selectively to the c-Rel subunit. Nat Immunol. 2007;8:984-991. 
23. Pappu BP, Lin X. Potential role of CARMA1 in CD40-induced splenic B cell proliferation and marginal zone B cell maturation. Eur J Immunol. 2006;36:3033-3043.

24. Oliver AM, Martin F, Gartland GL, Carter RH, Kearney JF. Marginal zone $\mathrm{B}$ cells exhibit unique activation, proliferative and immunoglobulin secretory responses. Eur J Immunol. 1997;27:2366-2374.

25. Hayden MS, Ghosh S. Signaling to NF-кB. Genes Dev. 2004; $18: 2195-2224$

26. Li Z, Wang H, Xue L, et al. Emu-BCL10 mice exhibit constitutive activation of both canonical and noncanonical NF- $\kappa B$ pathways generating marginal zone (MZ) B-cell expansion as a precursor to splenic MZ lymphoma. Blood. 2009;114:4158-4168.

27. Tusche MW, Ward LA, Vu F, et al. Differential requirement of MALT1 for BAFF-induced outcomes in B cell subsets. $J$ Exp Med. 2009;206:2671-2683.

28. Bhattacharyya S, Borthakur A, Tyagi S, et al. BCL10 is required for $\mathrm{NF}-\mathrm{\kappa B}$ nuclear translocation by both canonical and non-canonical pathways and for NF- $\kappa \mathrm{B}$-inducing kinase (NIK) phosphorylation. J Biol Chem. 2009; doi: 10.1974/jbc.M109.050815.

29. Amanna IJ, Dingwall JP, Hayes CE. Enforced bcl-xL gene expression restored splenic B lymphocyte development in BAFF-R mutant mice. J Immunol. 2003;170:4593-4600.

30. Lanier LL. NK cell recognition. Annu Rev Immunol. 2005;23:225-274.

31. Malarkannan S, Regunathan J, Chu H, et al. Bcl10 plays a divergent role in NK cell-mediated cytotoxicity and cytokine generation. J Immunol. 2007;179:3752-3762.

32. Rawlings DJ, Sommer K, Moreno-Garcia ME. The CARMA1 signalosome links the signalling machinery of adaptive and innate immunity in lymphocytes. Nat Rev Immunol. 2006;6:799-812.

33. Schulze-Luehrmann J, Ghosh S. Antigen-receptor signaling to nuclear factor KB. Immunity. 2006;25:701-715.

34. Tassi I, Cella M, Presti R, et al. NK cell-activating receptors require PKC-theta for sustained signaling, transcriptional activation, and IFNgamma secretion. Blood. 2008;112:4109-4116.

35. Sommer K, Guo B, Pomerantz JL, et al. Phosphorylation of the CARMA1 linker controls NF-KB activation. Immunity. 2005;23:561-574.

36. Matsumoto R, Wang D, Blonska M, et al. Phosphorylation of CARMA1 plays a critical role in $\mathrm{T}$ Cell receptor-mediated NF- $\kappa \mathrm{B}$ activation. Immunity. 2005;23:575-585.

37. Stilo R, Liguoro D, Di Jeso B, et al. Physical and functional interaction

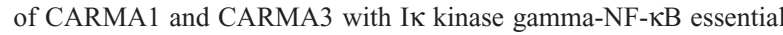
modulator. J Biol Chem. 2004;279:34323-34331.

38. Ishiguro K, Green T, Rapley J, et al. Ca2+/calmodulin-dependent protein kinase II is a modulator of CARMA1-mediated NF-KB activation. Mol Cell Biol. 2006;26:5497-5508.

39. Ishiguro K, Ando T, Goto H, Xavier R. Bcl10 is phosphorylated on Ser 138 by $\mathrm{Ca} 2+/$ calmodulin-dependent protein kinase II. Mol Immunol. 2007;44:2095-2100.

40. Brenner D, Brechmann M, Rohling S, et al. Phosphorylation of CARMA1 by HPK1 is critical for NF-kB activation in T cells. Proc Natl Acad Sci U S A. 2009;106:14508-14513.

41. Shinohara H, Maeda S, Watarai H, Kurosaki T. IкB kinase beta-induced phosphorylation of CARMA1 contributes to CARMA1 Bcl10 MALT1 complex formation in B cells. J Exp Med. 2007;204:3285-3293.

42. Wegener E, Oeckinghaus A, Papadopoulou N, et al. Essential role for I B kinase beta in remodeling Carma1-Bcl10-Malt1 complexes upon T cell activation. Mol Cell. 2006;23:13-23.

43. Lobry C, Lopez T, Israel A, Weil R. Negative feedback loop in T cell activation through I $\mathrm{KB}$ kinase-induced phosphorylation and degradation of Bcl10. Proc Natl Acad Sci U S A. 2007;104:908-913.

44. Bidere N, Ngo VN, Lee J, et al. Casein kinase 1alpha governs antigen-receptor-induced NF- $\mathrm{\kappa B}$ activation and human lymphoma cell survival. Nature. 2008

45. Tanner MJ, Hanel W, Gaffen SL, Lin X. CARMA1 coiled-coil domain is involved in the oligomerization and subcellular localization of CARMA1 and is required for $\mathrm{T}$ cell receptor-induced NF- $\mathrm{KB}$ activation. J Biol Chem. 2007;282:17141-17147.
46. Jun JE, Wilson LE, Vinuesa CG, et al. Identifying the MAGUK protein Carma-1 as a central regulator of humoral immune responses and atopy by genome-wide mouse mutagenesis. Immunity. 2003;18: 751-762.

47. Thome M. Multifunctional roles for MALT1 in T-cell activation. Nat Rev Immunol. 2008;8:495-500.

48. Sun L, Deng L, Ea CK, Xia ZP, Chen ZJ. The TRAF6 ubiquitin ligase and TAK1 kinase mediate IKK activation by BCL10 and MALT1 in T lymphocytes. Mol Cell. 2004;14:289-301.

49. Wu CJ, Ashwell JD. NEMO recognition of ubiquitinated Bcl10 is required for T cell receptor-mediated NF- $\mathrm{KB}$ activation. Proc Natl Acad Sci U S A. 2008;105:3023-3028.

50. Oeckinghaus A, Wegener E, Welteke V, et al. Malt1 ubiquitination triggers NF- $\mathrm{KB}$ signaling upon T-cell activation. Embo $J$. 2007;26:4634-4645.

51. Zhou H, Wertz I, O'Rourke K, et al. Bcl10 activates the NF-KB pathway through ubiquitination of NEMO. Nature. 2004;427:167-171.

52. Moreno-Garcia ME, Sommer K, Shinohara H, Bandaranayake AD, Kurosaki T, Rawlings DJ. MAGUK-controlled ubiquitination of CARMA1 modulates lymphocyte NF-KB activity. Mol Cell Biol. 2009.

53. Kojo S, Elly C, Harada Y, Langdon WY, Kronenberg M, Liu YC. Mechanisms of NKT cell anergy induction involve Cbl-b-promoted monoubiquitination of CARMA1. Proc Natl Acad Sci U S A. 2009; 106:17847-17851.

54. Scharschmidt E, Wegener E, Heissmeyer V, Rao A, Krappmann D. Degradation of Bcl10 induced by T-cell activation negatively regulates NF-אB signaling. Mol Cell Biol. 2004;24:3860-3873.

55. Hu S, Alcivar A, Qu L, Tang J, Yang X. CIAP2 inhibits anigen receptor signaling by targeting Bcl10 for degredation. Cell Cycle. 2006; $5: 1438-1442$.

56. Welteke V, Eitelhuber A, Duwel M, Schweitzer K, Naumann M, Krappmann D. COP9 signalosome controls the Carma1-Bcl10-Malt1 complex upon T-cell stimulation. EMBO Rep. 2009;10:642-648.

57. Sun SC. Deubiquitylation and regulation of the immune response. Nat Rev Immunol. 2008;8:501-511.

58. Duwel M, Welteke V, Oeckinghaus A, et al. A20 negatively regulates T cell receptor signaling to NF- $\mathrm{\kappa B}$ by cleaving Malt1 ubiquitin chains. $J$ Immunol. 2009;182:7718-7728

59. Uren AG, O'Rourke K, Aravind LA, et al. Identification of paracaspases and metacaspases: two ancient families of caspase-like proteins, one of which plays a key role in MALT lymphoma. Mol Cell. 2000;6:961-967.

60. Rebeaud F, Hailfinger S, Posevitz-Fejfar A, et al. The proteolytic activity of the paracaspase MALT1 is key in T cell activation. Nat Immunol. 2008;9:272-281.

61. Coornaert B, Baens M, Heyninck K, et al. T cell antigen receptor stimulation induces MALT1 paracaspase-mediated cleavage of the NF-KB inhibitor A20. Nat Immunol. 2008;9:263-271.

62. Salmena L, Lemmers B, Hakem A, et al. Essential role for caspase 8 in T-cell homeostasis and T-cell-mediated immunity. Genes Dev. 2003; $17: 883-895$.

63. Su H, Bidere N, Zheng L, et al. Requirement for caspase-8 in NF- $\mathrm{KB}$ activation by antigen receptor. Science. 2005;307:1465-1468.

64. Chau H, Wong V, Chen NJ, et al. Cellular FLICE-inhibitory protein is required for T cell survival and cycling. J Exp Med. 2005; 202:405-413.

65. Kawadler H, Gantz MA, Riley JL, Yang X. The paracaspase MALT1 controls caspase-8 activation during lymphocyte proliferation. Mol Cell. $2008 ; 31: 415-421$

66. Kataoka T, Tschopp J. N-terminal fragment of c-FLIP(L) processed by caspase 8 specifically interacts with TRAF2 and induces activation of the NF-kB signaling pathway. Mol Cell Biol. 2004;24:2627-2636.

67. Wang D, Matsumoto R, You Y, et al. CD3/CD28 costimulation-induced $\mathrm{NF}-\kappa \mathrm{B}$ activation is mediated by recruitment of protein kinase $\mathrm{C}$-theta, $\mathrm{Bcl10}$, and IKB kinase beta to the immunological synapse through CARMA1. Mol Cell Biol. 2004;24:164-171. 
68. Misra RS, Russell JQ, Koenig A, et al. Caspase-8 and c-FLIPL associate in lipid rafts with NF- $\mathrm{KB}$ adaptors during T cell activation. J Biol Chem. 2007;282:19365-19374.

69. Hara H, Bakal C, Wada T, et al. The molecular adapter Carma1 controls entry of IкB kinase into the central immune synapse. J Exp Med. 2004;200:1167-1177.

70. Medeiros RB, Burbach BJ, Mueller KL, et al. Regulation of NF- $\kappa B$ activation in $\mathrm{T}$ cells via association of the adapter proteins ADAP and CARMA1. Science. 2007;316:754-758.

71. Lee KY, D’Acquisto F, Hayden MS, Shim JH, Ghosh S. PDK1 nucleates $\mathrm{T}$ cell receptor-induced signaling complex for NF- $\mathrm{KB}$ activation. Science. 2005;308:114-118.

72. Park SG, Schulze-Luehrman J, Hayden MS, et al. The kinase PDK1 integrates $\mathrm{T}$ cell antigen receptor and CD28 coreceptor signaling to induce NF-кB and activate T cells. Nat Immunol. 2009;10:158-166.

73. Du MQ. MALT lymphoma: recent advances in aetiology and molecular genetics. J Clin Exp Hematop. 2007;47:31-42.

74. Holzmann K, Kohlhammer H, Schwaenen C, et al. Genomic DNA-chip hybridization reveals a higher incidence of genomic amplifications in pancreatic cancer than conventional comparative genomic hybridization and leads to the identification of novel candidate genes. Cancer Res. 2004;64:4428-4433.
75. Sanchez-Izquierdo D, Buchonnet G, Siebert R, et al. MALT1 is deregulated by both chromosomal translocation and amplification in B-cell non-Hodgkin lymphoma. Blood. 2003;101:4539-4546.

76. Dijkman R, Tensen CP, Jordanova ES, et al. Array-based comparative genomic hybridization analysis reveals recurrent chromosomal alterations and prognostic parameters in primary cutaneous large B-cell lymphoma. J Clin Oncol. 2006;24:296-305.

77. Shen L, Liang AC, Lu L, et al. Aberrant BCL10 nuclear expression in nasal NK/T-cell lymphoma. Blood. 2003;102:1553-1554.

78. Ngo VN, Davis RE, Lamy L, et al. A loss-of-function RNA interference screen for molecular targets in cancer. Nature. 2006;441:106-110.

79. Lenz G, Davis RE, Ngo VN, et al. Oncogenic CARD11 mutations in human diffuse large B cell lymphoma. Science. 2008;319:1676-1679.

80. Hailfinger S, Lenz G, Ngo V, et al. Essential role of MALT1 protease activity in activated B cell-like diffuse large B-cell lymphoma. Proc Natl Acad Sci U S A. 2009;106:19946-19951.

81. Kuo SH, Yeh PY, Chen LT, et al. Overexpression of B cell-activating factor of TNF family (BAFF) is associated with Helicobacter pyloriindependent growth of gastric diffuse large B-cell lymphoma with histologic evidence of MALT lymphoma. Blood. 2008;112:2927-2934.

\section{Publish your work in this journal}

The Journal of Blood Medicine is an international, peer-reviewed, open access, online journal publishing laboratory, experimental and clinical aspects of all topics pertaining to blood based medicine including but not limited to: Transfusion Medicine; Blood collection, Donor issues, Transmittable diseases, and Blood banking logistics; Immunohematology; Artificial and alternative

\section{Dovepress}

blood based therapeutics; Hematology; Biotechnology/nanotechnology of blood related medicine; Legal aspects of blood medicine; Historical perspectives. The manuscript management system is completely online and includes a very quick and fair peer-review system. Visit http://www.dovepress.com/ testimonials.php to read real quotes from published authors. 